Balasubramanian, Sriram

\section{Caenorhabditis elegans gene expression analysis using microarrays of covalently attached oligodeoxyribonucleotides}

\author{
Gary Hardiman, Yen Tran, Karin Franz-Bacon, Ralph Clover, \\ Robert W isotzkey, Abhijit Mazumder \& Sriram Balasubramanian
}

Axys Pharmaceuticals, 11099 N. Torrey Pines Rd., Suite 160, La Jolla, California 92037, USA

The largest genome to be completely sequenced to date is the nematode Caenorhabditis elegans. In such cases, the method of choice for expression analysis is to use oligonucleotide arrays designed directly from the genomic sequence. We have constructed such an oligonucleotide microarray with nearly 1,500 C. elegans oligonucleotide targets, which were synthesized with a 5 'amino group and then covalently immobilized on polymer-coated glass slides. Using this array, we have examined the developmental expression changes that occur during the life cycle of this organism by hybridizing them with fluorescently labelled cDNA generated from poly(A), as well as total RNA from various larval, embryonic, dauer and adult stages. When two sets of oligos for each gene were arrayed on the same chip, we found that the intensities were quite similar to each other, suggesting that one oligonucleotide per gene designed as described here is sufficient for differential gene expression analysis. This would enable densities of up to 9,216 genes per chip using our current system. The comparative, two-colour hybridizations provided a close look at many changes in the expression patterns during development, including the differential expression of certain cathepsin-like proteases. Expression levels determined by oligonucleotide microarrays were confirmed by northern blot as well as cDNA Southern blot using a nematode plasmid library. The advantages of this microarray system include being able to array PCR products at high densities, as demonstrated here by the observation of changes in expression pattern induced in HeLa cells by the cytokine interferon-gamma.

Baldocchi, Russ

\section{Oligonucleotide-array-based compara- tive genomic hybridization}

\author{
R.A. Baldocchi, R.J. Glynne, D. Kowbel, E. Tom, C. Collins, \\ D.H. Mack \& J.W. Gray \\ University of California at San Francisco Cancer Center, \\ San Francisco, California 94143-0808, USA \\ Eos Biotechnology, Inc., South San Francisco, California 94080, USA
}

Comparative genomic hybridization (CGH) provides a genome-wide scan that detects chromosomal regions which have copy number abnormalities (CNAs). For example, one can detect the loss of a region containing a tumour-suppressor gene or the amplification of one harbouring an oncogene. Typically, two genomes are compared by labelling total genomic DNA with different flourescent dyes and then co-hybridizing these complex probes to metaphase spreads. This enables mapping as well as semi-quantitative measurement of CNAs. Array CGH exploits contigued BACs (for example, BAC clones) as targets for increased resolution $(\sim 100 \mathrm{~kb})$ and increased quantitative capacity. In oligonucleotide-array CGH, a probe of reduced complexity and no repetitive elements is produced by making a PCR-based representation of each genomic DNA sample. In a 'proof-of-principle' approach, a multiplex PCR was performed for ten loci using primer pairs that contained gene-specific and adapter sequences. The target elements, printed on plastic slides, consisted of 50-mer oligonucleotides that mapped to the region flanked by primer sequences. This method requires less than $25 \mathrm{ng}$ of starting genomic DNA template. The normalized copy number measurements shown below are similar to those obtained using the well-established FISH methodology, by which the amplifications of ZNF217 and AIBC1 in MCF7 and CCND1 in 600MPE were clearly detected, as was a loss of TP53 in 600MPE. This consistancy supports the feasibility of this approach.

\begin{tabular}{llllllllll}
\hline \multicolumn{2}{l}{ Relative Genomic Copy Number $( \pm$ SD) } & & & & & \\
Genome: Loci: & & & & & & & & \\
& $\begin{array}{l}\text { BRCA2 } \\
\text { CCND1 }\end{array}$ & DCC & ERBB2 & MYC & AIB1 & P53 & ZNF217 & \\
ZNF218 & & & & & & & & & \\
MCF7 & $1.0 \pm 0.1$ & $1.0 \pm 0.1$ & $1.1 \pm 0.1$ & $0.8 \pm 0.2$ & $2.0 \pm 0.1$ & $17.3 \pm 2.3$ & $0.7 \pm 0.1$ & $9.7 \pm 0.3$ & $1.2 \pm 0.1$ \\
600MPE & $1.8 \pm 0.4$ & $6.7 \pm 0.4$ & $2.2 \pm 0.2$ & $1.8 \pm 0.4$ & $1.2 \pm 0.2$ & $1.3 \pm 0.2$ & $0.6 \pm 0.1$ & $2.1 \pm 0.2$ & $1.1 \pm 0.1$ \\
\hline
\end{tabular}

Barrett, Michael T.

\section{High yields of RNA and DNA suitable for array analysis from cell sorter purified epithelial cell and tissue populations}

\author{
M.T. Barrett, J. Glogovac, P. Porter, B.J. Reid \& P.S. Rabinovitch \\ Fred Hutchinson Cancer Research Center, 1100 Fairview Avenue N., P.O. Box \\ 19024, Seattle, Washington 98109-1024, USA
}

INTRODUCTION: Microarray technologies have made it possible to perform high-throughput and comprehensive analyses of nucleic acid sequences and expression. However, the technology to efficiently obtain high yields of RNA and DNA suitable for array analysis from purified populations of neoplastic cells from human tissues has not been well addressed. Microdissection can enrich populations of cells present in various tumour tissues, however it is not easily automated for high throughput use. In addition, there are some tissues in which it is difficult to separate neoplastic from contaminating normal cells. We have previously shown that DNA suitable for high throughput genotyping can be obtained from nuclei of neoplastic cell populations purified by flow cytometric cell sorting. To apply array technology to in vivo studies of human neoplastic progression, we have developed protocols for rapid and efficient isolation of RNA and DNA from flow-cytometrically purified whole epithelial cells from fresh and frozen primary tissue. We have also utilised a real-time PCR assay to determine RNA quality after flow sorting. MATERIALS AND METHODS: A549 lung adenocarcinoma cells were cultured in RPMI supplemented with 5\% FCS. Cells were trypsinised according to standard protocols, resuspended as single cells in the aqueous reagent RNAlater ${ }^{\mathrm{TM}}$ (Ambion, Inc. Austin TX) and kept on ice. Breast tissues were obtained from mastectomy samples and biopsies frozen in minimal essential media (Gibco, Rockville MD) containing 10\% dimethyl sulfoxide (Fisher, Fair Lawn, NJ). Single-cell suspensions were created by placing fresh or thawed tissues in phosphate buffered saline with $1 \%$ bovine serum albumin (BSA; PBA), and mincing. The minced tissue was resuspended in PBA, filtered through $40 \mathrm{u}$ mesh, centrifuged then resuspended in 100 ul of RNAlater ${ }^{\mathrm{TM}}$. Single-cell suspensions were stained with epithelial-specific cytokeratin CAM 5.2-FITC (Becton Dickinson, San Jose, CA) or a FITC-labeled isotype matched mouse immunoglobulin IgG2a-FITC (Becton Dickinson, San Jose, CA) on ice for 1 hour. Samples were diluted with $1.5 \mathrm{ml}$ of PBA then resuspended in $0.5-1.0 \mathrm{ml}$ of 10 $\mathrm{g} / \mathrm{ml}$ 2,4 diamidino-6-phenylindole (DAPI) in NST buffer ( $146 \mathrm{mM} \mathrm{NaCl}, 10 \mathrm{mM}$ Tris base, $0.1 \%$ NP40 (Sigma), $2 \mathrm{mM} \mathrm{Ca}, 20 \mathrm{mM} \mathrm{Mg}$, and $0.05 \%$ BSA (Sigma), $\mathrm{pH}$ 7.4). All centrifugation steps were performed at $4 \mathrm{x} \mathrm{g}$ for 8 minutes at $2 \mathrm{C}$ and all reagents were DNAse/RNAse free. Whole cells were sorted by DNA content and cytokeratin staining intensity on a Becton Dickinson Vantage flow cytometer, with $100 \mathrm{~mW}, 488 \mathrm{~nm}$ excitation (FITC and PI) and $100 \mathrm{~mW}, 354-361 \mathrm{~nm}$ (UV) excitation (DAPI). The instrument was aligned daily using "DNA check" beads (Coulter, Miami, Fl.) to give coefficients of variation of $<3 \%$. List-mode data were analysed using MultiCycle AV and MultiPlus AV software (Phoenix Flow Systems, San Diego, CA). RNA was isolated according to standard protocols, while DNA was simultaneously extracted from organic phases. The integrity of RNA from flow sorted cells was determined by real-time PCR assays, TaqMan ${ }^{\circledR}$ 